\title{
Dental Stem Cells: Sources and Potential Applications
}

\author{
Su-Min Lee • Qunzhou Zhang • Anh D. Le
}

Published online: 30 January 2014

(C) Springer International Publishing AG 2014

\begin{abstract}
In recent years, stem cell research in dentistry has grown rapidly with the potential application for oral and maxillofacial tissue regeneration. Mesenchymal stem cells (MSCs) from the oral and maxillofacial region are easy to access, have a high proliferation rate, multipotency, and potent immunomodulatory functions. They are excellent cell sources not only for stem cell-based therapy of dental and craniofacial diseases, but also with the potential for the treatment of other inflammatory diseases. In this review, we provide an overview of different types of MSCs that have been isolated and characterized from several origins such as dental pulp, exfoliated deciduous teeth, the periodontal ligament, the dental follicle, the dental papilla, oral mucosa, and gingiva, with the focus on the potential clinical applications for each type of dental stem cell.
\end{abstract}

Keywords MSCs Mesenchymal Stem Cells · DSCs Dental Stem Cells · DPSCs Dental Pulp Stem Cells · SHED Stem Cells From Human Exfoliated Deciduous Teeth · SCAP Stem Cells From Root Apical Papilla · PDLSCs Periodontal Ligament Stem Cells · DFSCs Dental Follicle Stem Cells · GMSCs Gingiva-Derived Mesenchymal Stem/Stromal Cells . Multipotency $\cdot$ Immunomodulation $\cdot$ Inflammatory Disease

S.-M. Lee

Department of Endodontics, Penn Dental Medicine of the University of Pennsylvania, Philadelphia, PA 19104, USA

e-mail: suminlee@dental.upenn.edu

Q. Zhang $\cdot$ A. D. Le

Department of Oral and Maxillofacial Surgery and Pharmacology, Penn Dental Medicine and Penn Medicine Hospital of the University of Pennsylvania, Philadelphia, PA 19104, USA

\section{A. D. Le $(\square)$}

Department of Oral \& Maxillofacial Surgery, University of

Pennsylvania School of Dental Medicine, 240 South 40th Street, Philadelphia, PA 19104, USA

e-mail: anhle@dental.upenn.edu

\section{Introduction}

Stem cells are defined as a special group of clonogenic cells that are characterized by the ability for self-renewal and multilineage differentiation. These cells are responsible for normal tissue renewal as well as for healing and regeneration after injuries [1]. Stem cells can be divided into three main types, embryonic stem cells (ESCs), adult or postnatal stem cells (ASCs), and induced pluripotent stem cells (iPSCs). ESCs are found in the inner cell mass of mammalian blastocysts during the early stages of embryo development and have unlimited expansion and pluripotency to differentiate into all types of somatic cells [2]. A new source of pluripotent stem cells, iPSCs, have been innovatively generated from human somatic cells and potentially can provide tremendous opportunities for tissue regeneration and patient-specific therapies, while avoiding ethical issues surrounding the use of human embryos and tumorigenic potential $[3,4]$. ASCs have been identified in almost all the postnatal tissues. Because of their easy accessibility and great ability to generate a tissue different to the site from their origin, ASCs have increasing potential to be used for treatment of degenerative diseases [5]. Mesenchymal stem cells (MSCs) are multipotent progenitor cells present in many tissues throughout the body and have the capacity to differentiate into bone, cartilage, tendon, fat, and muscle [6]. Furthermore, MSCs are important immunoregulatory cells because they can sense and control inflammation in injured tissues by expressing a variety of chemokines and cytokines [7]. To date, different types of MSCs have also been isolated and characterized from oral and maxillofacial regions. The objective of this review is to describe new findings in the field of dental stem cell research and their potential applications in tissue regeneration and therapy of inflammation-related diseases.

\section{Dental Stem Cells (DSCs)}

Sources of Stem Cells from the Oral-Maxillofacial Regions

Several types of dental MSCs have been identified in different dental tissues, including: 1) the dental pulp of permanent teeth 
(dental pulp stem cells, DPSCs) [8••]; 2) the naturally exfoliated deciduous teeth (stem cells from human exfoliated deciduous teeth, SHED) [9]; 3) the apical papilla (stem cells from apical papilla, SCAP) $[10,11]$; 4) the periodontal ligament (periodontal ligament stem cells, PDLSCs) [12]; 5) the dental follicle (dental follicle progenitor cells, DFPCs) [13], and 6) the oral mucosa and gingiva (gingiva-derived mesenchymal stem/stromal cells, GMSCs) [14••]. In comparison to bone marrow-derived mesenchymal stem cells (BM-MSCs), the most extensively studied MSCs [15], these DSCs, derived from oral and maxillofacial areas, have also been demonstrated to possess potent self-renewal/colony forming and multipotent differentiation capabilities (Fig. 1).

\section{Dental Pulp Stem Cells (DPSCs)}

MSC populations have been isolated from dental pulp tissue of permanent human teeth, and are generally referred to as DPSCs. DPSCs fulfill the criteria as stem cells, based on their selfrenewal and the ability to differentiate into osteoblasts, adipocytes, neural cells, and odontoblasts $[8 \cdot \bullet, 15]$. Additional studies have confirmed that DPSCs can also differentiate into chondrocytes and myoblast-like cells and induce endogenous axon guidance $[5,16,17]$. Subsequent studies have demonstrated that transplantation of in vitro expanded DPSCs mixed with hydroxyapatite-tricalcium phosphate (HA/TCP) scaffolds can generate dentin/pulp-like complexes in immunocompromised mice $[8 \bullet \bullet, 15,18]$. The newly formed vascularized pulp-like tissue is surrounded by a layer of odontoblast-like cells that express dentin sialophosphoprotein (DSPP) and contains dentinal tubules $[8 \bullet \bullet, 19]$. The differentiation of DPSCs to a specific cell lineage is mainly determined by the components of local microenvironment such as growth factors, signaling molecules, transcription factors and extracellular matrix proteins. The odontoblastic differentiation of DPSCs and formation of reparative dentin over the exposed pulp are promoted by dentin matrix protein-1 (DMP-1) [20]. Additionally, DPSCs can be induced into odontoblast lineage when treated with transforming growth factor- $\beta 1$ (TGF- $\beta 1)$ alone or in combination with fibroblast growth factor-2 (FGF-2) [21].

\section{Clinical Application}

\section{Dentin-Pulp Tissue Regeneration}

It has been considered a difficult task to regenerate all of the pulp-dentin tissue due to conditions whereby the pulp tissue usually has only one apical foramen to allow angiogenesis for the engineered tissue, and there are different types of cells in different zones with complex innervation [22,23]. A study using human tooth root fragments with an enlarged canal space showed that the regenerated pulp-dentin-like tissue formed a continuous layer with original dentin surface when the canal was filled with PLG scaffold seeded with DPSCs [24]. Most recently, complete pulp regeneration was achieved in adult canine pulpectomized teeth after autologous pulp stem/progenitor $\left(\mathrm{CD} 105^{+}\right)$cells with stromal cell-derived factor-1 (SDF-1) were transplanted into a root canal [25•]. Furthermore, functional
Fig. 1 Sources of dental stem cells and their multilineage differentiation capability and potential clinical applications for regenerative and immunomodulatory therapy. DPSCs: dental pulp stem cells [8••]; SHED: stem cells from exfoliated deciduous teeth [9]; SCAP: stem cells from apical papilla $[10,11]$; PDLSCs: periodontal ligament stem cells [12]; DFPCs: dental follicle progenitor cells [13]; GMSCs: gingiva-derived mesenchymal stem/stromal cells $[14 \bullet \bullet]$

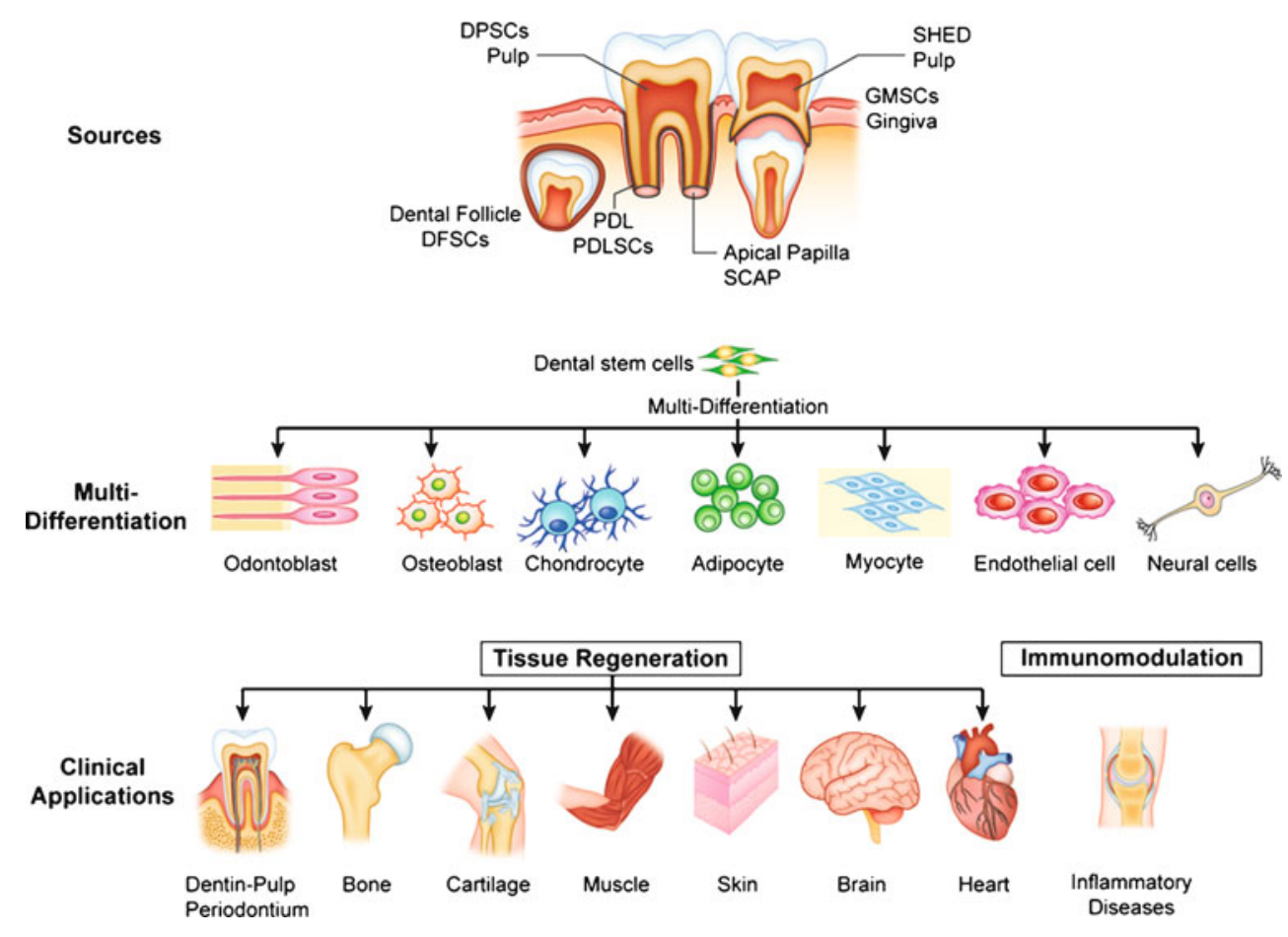


angiogenesis/vasculogenesis and reinnervation/neurogenesis in the regenerated pulp tissue have been demonstrated through laser Doppler analysis and the electric pulp test [26]. Although these studies have primarily shown the potential of complete pulp regeneration, they were done under the conditions that are not relevant to most clinical scenarios with infected root canals and apical periodontitis. Under these experimental conditions, cells/scaffold were implanted into freshly removed clinically healthy pulp tissues in incisors with large apical foramina. Therefore, further studies are needed to test the limit of the canal opening size that allows pulp regeneration in the coronal end of the canal and whether the addition of angiogenic factors can compensate for the smaller canal opening [24].

Dentin-Pulp Tissue Regeneration with Inflamed Pulp Tissue

Clinically, when a tooth has been diagnosed with irreversible pulpitis, the available pulp is considered inflamed and/or infected and, therefore, it is removed even though some portion of the pulp is still viable. Recent studies have compared the cells from normal and inflamed human dental pulps regarding the presence of stem cells, their proliferation and differentiation potentials [27-30]. Diseased pulp contains viable cells with the potential for ex vivo expansion and proliferation, a similar percentage of STRO-1-positive cells and ex vivo odonto-osteogenic differentiation capacity. As the inflamed pulp contains preserved stem cells, it could be envisaged as a suitable source of DPSCs for dental pulp regeneration, not simply discarded as biological waste.

\section{Periodontal Regeneration}

Recent studies have demonstrated that implantation of DPSCs into periodontal defects of dogs results in the regeneration of well-formed mature bone with neovascularization [31,32]. One clinical study in humans tested to treat alveolar bone defects with autologous DPSCs, which were obtained from maxillary third molars and then combined with a collagen sponge scaffold. The filling of this biocomplex in the space left by extraction of the mandibular third molar resulted in the complete regeneration of defective bone at the injury site, suggesting DPSCs as a potential cell source for periodontal regeneration [33].

\section{Application for Non-Oral Tissue Regeneration}

Because of their versatility, DPSCs have been shown to possess therapeutic effects for myocardial infarction [34], muscular dystrophy [35], cerebral ischemia, and chemical-induced corneal injury [36, 37]. In a recent study, neuronally predifferentiated DPSCs were labeled with a vital cell dye and transplanted into the cerebrospinal fluid of rats with induced cortical lesions. Those cells were subsequently detected in the injured cortex and found to express neuron- specific markers, suggesting that engrafted DPSC-derived cells can integrate into the host brain and may serve as a useful source of neuro- and gliogenesis in vivo [38]. Moreover, the transplantation of DPSCs promoted neurogenesis and vasculogenesis in an induced peri-infarct area, and enhanced recovery after TMCAO in rats, indicating that DPSCs can be a potential candidate in the treatment of stroke [39].

\section{Stem Cells from Human Exfoliated Deciduous Teeth (SHED)}

SHED were first identified and isolated noninvasively from the pulp of human exfoliated primary incisors, which are considered disposable tissues [9]. A subsequent study also isolated and classified SHED as being 'immature DPSCs' (IDPSCs). These cells expressed the embryonic stem cell markers Oct4, Nanog, stage specific embryonic antigens (SSEA-3, -4), and tumor recognition antigens (TRA-1-60 and TRA-1-81) [40]. SHED can undergo osteogenic, adipogenic, neurogenic, myogenic, chondrogenic, endothelial, and odontoblastic differentiation $[9,40-43]$. In comparison with DPSCs, SHED exhibit faster proliferation rates and increased population doublings [8••, 41, 44]. Although both SHED and DPSCs originate from the dental pulp, they demonstrate differences regarding their odontogenic differentiation and osteogenic induction. Ex vivo expanded SHED transplanted into immunocompromised mice generate odontoblast-like cells directly associated with a dentin-like structure. However, they are unable to regenerate complete dentinpulp-like complexes in vivo $[8 \bullet \bullet, 9]$. On the contrary, DPSCs are able to form dentin-pulp-like complexes, but not lamellar bone $[8 \bullet \bullet]$. The levels of alkaline phosphatase activity and osteocalcin production during osteogenic differentiation are higher for SHED than for DPSCs [45]. Furthermore, SHED may present an osteoinductive potential since they were able to induce differentiation of recipient murine cells into bone-forming cells [9]. These discrepancies in the properties of DPSCs and SHED may be related to their different gene expression profiles, as the genes related to cell proliferation and extracellular matrix formation, such as TGF- $\beta$, FGF-2, collagen (Col) I, and Col III, were significantly highly expressed in SHED compared with DPSCs [44]. The potential use of SHED as cell-based therapies has expanded to a large number of tissues due to their high degree of plasticity, stemness, and ability to cross lineage boundaries.

\section{Clinical Application}

\section{Dentin-Pulp Regeneration}

Using a tooth slice model, SHED seeded onto the synthetic scaffolds form well-vascularized pulp-like tissue in vivo. In addition, odontoblast-like cells derived from this tissue 
express dentin sialoprotein (DSP) and localize against the existing dentin surface [43]. A subsequent study using the same model demonstrated that SHED differentiated into functional odontoblasts capable of generating tubular dentin and angiogenic endothelial cells [41]. Notably, when BMP-2 signaling was blocked, SHED could not differentiate into odontoblastic-like cells in tooth slice/scaffolds [42]. Most recently, a closer clinical model using extracted endodontically treated premolars was designed to test the possibility of pulp tissue regeneration by implanting SHED seeded on two different scaffolds (polylactic acid and collagen) incorporated with different types of growth factors (BMP-2, TGF- $\beta 1$ ). An ultrastructural examination of the SEM micrographs revealed cell adherence within all of the pulp constructs, with little difference between the scaffold types or the addition of growth factors. These findings suggest that future regenerative endodontic treatment may involve the cleaning and shaping of root canals followed by the implantation of vital pulp tissue constructs created in the laboratory [46].

\section{Craniofacial Bone Regeneration}

SHED combined with HA/TCP can repair critical-size calvarial defects in mice with substantial bone formation [47]. Furthermore, SHED have been shown to regenerate bone in critical-size mandibular defects in mini pigs. This result from a pre-clinical study in a large animal model may permit translation of stem cell-based therapy in orofacial reconstruction to human clinical trials [48]. Embryologically derived from the neural crest cells, SHED may share similar tissue origin with the mandibular bone cells, and, therefore, may serve as a better cell source for the regeneration of alveolar and orofacial bone defects.

\section{Neural Tissue Regeneration}

SHED have been induced to express neural markers such as nestin [49] and form neural-like spheres with a medium optimized for neural stem cells in vitro. After transplantation of SHED spheres into parkinsonian rats, their behavioral disorders were alleviated, suggesting SHED may be a promising source for the treatment of neurodegenerative diseases [50].

\section{Stem Cells from Root Apical Papilla (SCAP)}

The apical papilla refers to the tissue loosely attached to the apex of the developing root in human immature permanent teeth [10]. There is a cell-rich zone lying between the apical papilla and the dental pulp. The distinction between these tissues is that the apical papilla represents a precursor tissue for the radicular pulp and plays a pivotal role in root development $[10,11,51]$. The MSC surface marker STRO-1 was positive in apical papilla cells, which led to the discovery of new type of stem cells in this tissue [11]. SCAP have the capacity to undergo osteo/dentinogenic, neurogenic, and adipogenic differentiation, and can also differentiate into odontoblast-like cells and produce dentin-like tissue in both in vitro and in vivo models [11]. Compared with DPSCs, SCAP display an expression pattern of osteo/dentinogenic markers and growth factor receptors similar to that observed in DPSCs, but these markers are expressed at lower levels in SCAP than in DPSCs. As SCAP are derived from a developing tissue, they are more robust than DPSCs in terms of population doubling capacity, proliferation rate, telomerase activity, and cell migration ability $[11,51]$. All of these findings suggest that SCAP may represent a population of early stem/progenitor cells and are suitable for cell-based tissue regeneration, particularly preferential for root regeneration $[11,51,52]$.

\section{Clinical Application}

\section{Dentin/Bone (Hard Tissue) Regeneration}

SCAP represent a population of cells participating in root formation and are able to differentiate into odontoblast-like cells with a very active cellular migratory and mineralization potential, leading to organized dentin-like structures in vitro. These structures progressively express differentiation markers, including DSPP, BSP, OCN, ALP, thus showing the characteristics of osteodentin [52]. When SCAP are combined with HA scaffolds and implanted subcutaneously in immunocompromised rats, bone- and dentin-like mineralized tissues are formed within 12 weeks following transplantation [53]. Furthermore, when SCAP were seeded onto synthetic scaffolds, inserted into tooth fragments, and then transplanted into immunodeficient mice, a pulp-like tissue with wellestablished vascularity was induced and a continuous layer of dentin-like tissue was deposited onto the canal dentinal wall [24]. These findings have provided evidence that SCAP can be used in tissue engineering/regeneration protocols as an approachable stem cell source for osteo/odontogenic differentiation and biomineralization.

\section{Continued Root Formation and Bio-Root Engineering}

Interestingly, when the apical papilla was removed at an early stage of the root development in mini pigs, the root development was halted despite the pulp tissue being intact. In contrast, other roots containing apical papilla showed normal growth [51]. Furthermore, several clinical cases also demonstrated the role of apical papilla in root formation. During the treatment for a fractured immature incisor with pulp exposure, the apical papilla was retained while the pulp was extirpated. 
Continued root-tip formation was observed after root canal treatment [51]. After root canal disinfection, surviving SCAP may give rise to primary odontoblasts to complete root formation in several cases of apexogenesis in an infected immature tooth with periradicular periodontitis or abscess $[23,51$, $54,55]$. This approach may be more conservative and should be provided when treating immature teeth with endodontic infection. Collectively, these findings support that the apical papilla is likely to play a pivotal role in root formation. In a mini pig model, the root-shaped HA/TCP block containing SCAP was coated with Gelfoam containing PDLSCs and inserted into the alveolar socket of a recently extracted tooth. After 3 months, a bio-root was formed and capable of supporting a porcelain crown and participating in normal tooth function. This result implies the feasibility of using a combination of autologous SCAP/PDLSCs in conjunction with artificial dental crowns for functional tooth regeneration [11].

\section{Periodontal Ligament Stem Cells (PDLSCs)}

The periodontal ligament (PDL) is a specialized tissue located between the cementum and alveolar bone and plays an important role in tooth support and in regulating alveolar bone volume. The presence of heterogeneous cell populations within the PDL suggests that this tissue contains progenitor cells which maintain tissue homeostasis and the regeneration of the periodontium. PDLSCs have been demonstrated to be a population of clonogenic adherent cells with the characteristics of postnatal stem cells and express cementoblastic and osteoblastic markers [12]. Similar to the other DSCs, PDLSCs under defined culture conditions can differentiate into osteogenic, adipogenic, and chondrogenic cells [56-58]. PDLSCs have also been shown to differentiate into neuronal precursors [59]. Interestingly, the multipotent differentiation capability of PDLSCs was up-regulated by Hertwig's epithelial root sheath cells in vitro [60]. When PDLSCs were transplanted into immunocompromised mice, cementum/PDL-like structures were regenerated [12].

\section{Clinical Application}

\section{Periodontal Regeneration}

Since PDLSCs were firstly demonstrated to repair periodontal defects in mandible of rats [12], extensive research has followed to explore the potential of using PDLSCs to treat periodontal diseases in various larger animal models. Autologous PDLSCs from miniature swine were expanded ex vivo and labeled with green fluorescent protein (GFP). Afterwards, these cells combined with HA-TCP were transplanted into periodontal defects and GFP-labeled cells were identified in the newly formed bone with regenerated cementum and PDL in defect areas [61]. For more challenging periodontal defects, apical defects were created in dogs to simulate human advanced periodontitis where one of the tooth roots was isolated from the surrounding bone. When ex vivo expanded PDLSCs, DPSCs, and DFSCs were grafted into these defects without scaffold, PDLSCs showed the best regenerating capacity of periodontium as well as peripheral nerve and blood vessel [62]. Besides animal models, a retrospective pilot study in human has also demonstrated the therapeutic potential of autologous periodontal ligament progenitor cells (PDLPs) obtained from wisdom teeth. PDLPs behaved as PDLSCs, although they did not express the same panel of markers and showed lower multiple differentiation potential in vitro. PDLPs were implanted with bone grafting material into intrabony defects of patients who were monitored for 32 to 72 months. All defects were reconstructed and improved clinical outcomes were observed specifically in decreased tooth mobility and probing depth [63•]. Moreover, PDLSCs have been used to establish treatments associated with titanium dental implants. PDL regeneration was detected on titanium implant surfaces in a rat molar implant model [64]. When PDLSCs loaded HA/TCP were implanted in a canine saddlelike peri-implant defect model, newly formed bone was found to fill in defects [65]. In addition, one human clinical study has demonstrated cell-coated implant placement in the mandible and PDL formation around the implant surface. This model, so-called ligaplants, combined PDL cells with implant biomaterial and was successfully functioning during $6-60$ months of observation [66]. These findings hold great potential for PDLSCs as an alternative approach to osseointegrated dental implants and may provide efficient methods to enhance the outcome for implant treatment.

\section{Dental Follicle Precursor Cells (DFPCs)}

The dental follicle is a loose ectomensenchyme-derived connective tissue that surrounds the developing tooth. This tissue contains progenitors for PDL cells, cementoblasts and osteoblasts, and then may play a crucial role in tooth development. Precursor cells have typically been isolated from human dental follicles of impacted third molars and express stem cell markers Notch1, STRO-1, and nestin [13]. DFPCs have the ability to differentiate into osteoblasts/cementoblasts, chondrocytes and adipocytes in vitro $[13,67,68]$. DFPCs can also form compact calcified nodules in vitro, suggesting that these cells can differentiate into cementum [13]. In addition, after transplantation into immunodeficient mice, DFPCs could form fibrous tissues and a cementum-like matrix on the surface of HA beads $[69,70]$ and show osteogenic potential [71]. Meanwhile, the expression of cell surface markers compatible with those of fibroblasts was confirmed in human dental follicle tissues, suggesting the 
presence of immature PDL fibroblasts [72]. Furthermore, in vivo study showed that DFPCs have the capacity to generate PDL [73]. DFPCs, in common with SCAP, represent a population of cells from a developing tissue and might thus exhibit a greater plasticity than other DSCs. Interestingly, when comparing DFPCs and SCAP at the crown-forming stage, both cells shared similar characteristics and were capable of forming hard tissue in vivo as well as in vitro. However, SCAP had a significantly higher calcium accumulation than that in DFPCs and expressed an odontoblast marker, whereas DFPCs expressed a cementoblast marker. These differences might be explained by the developmental stage of the tooth germs from which these cells are derived [74]. Both DFPCs and SHED can differentiate into neuron-like cells, but showed different expression patterns of neural cell markers when the cells were grown under the same culture conditions [75].

\section{Clinical Application}

\section{Tooth Root Regeneration}

When DFPCs in combination with a treated dentin matrix were implanted in the alveolar fossa of rats, they generated root-like tissues with a pulp-dentin complex and a PDL connecting a cementum-like layer to host alveolar bone, but not in the skull and omental pockets. These results indicate that the microenvironment into which stem cells are implanted affects the capacity of these cells to regenerate differing tissues. Furthermore, these findings demonstrate that DFPCs can be a feasible cell source for tooth roots regeneration [68].

\section{Gingiva-Derived Mesenchymal Stem/Stromal Cells (GMSCs)}

Gingiva is a unique fibrous tissue that lies over the alveolar bone and surrounds the teeth, recognized as a biological mucosal barrier and a distinct component of the oral mucosal immunity. Wound healing within the gingiva and oral mucosa exhibit markedly reduced inflammation, rapid wound closure, and fetal-like scarless healing [76, 77]. Among all dental tissues, gingiva is the most easily accessible and harvested. GMSCs have been isolated from the lamina propria layer of human gingiva and are shown to express pluripotency-related markers such as Oct-4, SSEA-4 and STRO-1 [14••, 78]. GMSCs have self-renewal capability and can differentiate into osteoblasts, adipocytes, chondrocytes, and neural cells [14••, 78-81]. GMSCs showed highly clonogenic and longterm proliferative capability while they retain stable morphology and MSC characteristic at higher passages [78]. These properties highlight GMSC as highly attractive candidate cells for clinical application wherein a large scale of ex vivo cell expansion is necessary. In vivo, GMSCs embedded with carriers and transplanted into immunocompromised mice can generate connective tissue-like structures [14••, 78], bone matrix [80, 82], and even two germ layer-derived (teratoma-like) tissues [81].

\section{Clinical Application}

\section{Wound Healing}

When GMSCs are seeded on type I collagen and implanted locally, they can significantly promote the repairing of mandibular and the critical-sized calvarial defects in rats in 2 months [80]. In a murine excisional full-thickness skin wound model, systemic infusion of GMSCs significantly promoted the repair process as shown by rapid re-epithelialization and increased angiogenesis [83]. In addition, systemic infusion of GMSCs could also mitigate chemotherapeutic drug-induced oral mucositis in mice as presented by reversal of body weight loss, restoration of the disrupted epithelial lining, and increased proliferation of basal cells [84].

\section{Immunomodulatory Therapies for Inflammatory Diseases}

Recently, accumulating evidence supported that MSCs are powerful modulators of both the innate and adaptive immune responses. These findings explain that the beneficial effect of MSCs for tissue regeneration is specifically through interaction with host/resident cells and production of a large array of secretory immunomodulatory factors $[85,86]$. Systemic administration of GMSCs in murine colitis could reverse body weight loss, improve the overall colitis score, and restore normal intestinal architecture [14••]. The clinical signs of contact hypersensitivity, an allergy-related inflammatory disease, were alleviated after prophylactic and therapeutic administration of GMSCs. It has been shown that GMSC treatment reduced the infiltration of dendritic cells, $\mathrm{CD} 8^{+} \mathrm{T}$ cells, Th17, total and degranulated mast cells, and decreased the level of a variety of inflammatory cytokines [87]. Furthermore, systemic infusion of GMSCs exhibited remarkable immune tolerance and promoted the survival of skin allografts with an increased infiltration of Tregs [78]. Most recently, the promising therapeutic effect of GMSCs was demonstrated in an experimental collagen-induced arthritis model. Infusion of GMSCs significantly reduced the severity of arthritis and down-regulated the production of inflammatory cytokines (interferon- $\gamma$ and interleukin-17A) [88]. These findings support immunomodulatory effects of GMSCs and their interplay with innate and adaptive immune cells as well as their potential clinical applications in the treatment of several inflammation-related disease models [14••, 78, 83, 84, 87, 88]. 


\section{Conclusion}

Mesenchymal DSCs derived from oral and maxillofacial regions are easily accessible multipotent cells that can be noninvasively isolated and differentiated. Accumulating findings from various in vivo and in vitro studies provide hope for future applications of DSCs in regenerative dentistry and systemic degenerative diseases. In particular, as DSCs possess immunomodulatory capabilities to intervene both innate and adaptive immunity, their use for treating graft-vs.-host disease (GvHD), immune- and inflammation-related diseases holds great promise. However, several issues still need to be resolved, including the ex vivo expansion, differentiation efficiency, and safety in the use of DSCs. In addition, reliable ways to control previously inflammatory environments need to be established before the application of DSCs for the purpose of tissue regeneration. Further studies should be performed to clarify the underlying mechanisms of regeneration of the lost tissues and immunomodulatory properties of DSCs and then move the field forwards to human clinical trials.

Acknowledgments This work was supported by National Institute of Health Research Grant, R01DE 019932, Oral and Maxillofacial Surgery Foundation (OMSF) Research Grant.

\section{Compliance with Ethics Guidelines}

Conflict of Interest Dr. Su-Min Lee and Dr. Qunzhou Zhang declare this work was supported by National Institute of Health Research Grant, R01DE 019932, Oral and Maxillofacial Surgery Foundation (OMSF) Research Grant.

Dr. Anh D. Le declares this work was supported by National Institute of Health Research Grant, R01DE 019932, Oral and Maxillofacial Surgery Foundation (OMSF) Research Grant.

Human and Animal Rights and Informed Consent This article does not contain any studies with human or animal subjects performed by any of the authors.

\section{References}

Papers of particular interest, published recently, have been highlighted as:

- Of importance

-. Of major importance

1. van der Kooy D, Weiss S. Why stem cells? Science. 2000;287 (5457):1439-41.

2. Thomson JA, Itskovitz-Eldor J, Shapiro SS, et al. Embryonic stem cell lines derived from human blastocysts. Science. 1998;282 (5391):1145-7.

3. Takahashi K, Yamanaka S. Induction of pluripotent stem cells from mouse embryonic and adult fibroblast cultures by defined factors. Cell. 2006;126(4):663-76.
4. Okita K, Nakagawa M, Hyenjong H, Ichisaka T, Yamanaka S. Generation of mouse induced pluripotent stem cells without viral vectors. Science. 2008;322(5903):949-53.

5. Arthur A, Shi S, Zannettino AC, et al. Implanted adult human dental pulp stem cells induce endogenous axon guidance. Stem Cells. 2009;27(9):2229-37.

6. Prockop DJ. Marrow stromal cells as stem cells for nonhematopoietic tissues. Science. 1997;276(5309):71-4.

7. Newman RE, Yoo D, LeRoux MA, Danilkovitch-Miagkova A. Treatment of inflammatory diseases with mesenchymal stem cells. Inflamm Allergy Drug Targets. 2009;8(2):110-23.

8.• Gronthos S, Mankani M, Brahim J, Robey PG, Shi S. Postnatal human dental pulp stem cells (DPSCs) in vitro and in vivo. Proc Natl Acad Sci U S A. 2000;97(25):13625-30. This is the first study demonstrating that MSC can be isolated from the dental pulp tissue.

9. Miura M, Gronthos S, Zhao M, et al. SHED: stem cells from human exfoliated deciduous teeth. Proc Natl Acad Sci U S A. 2003;100 (10):5807-12.

10. Sonoyama W, Liu Y, Yamaza T, et al. Characterization of the apical papilla and its residing stem cells from human immature permanent teeth: a pilot study. J Endod. 2008;34(2):166-71.

11. Sonoyama W, Liu Y, Fang D, et al. Mesenchymal stem cellmediated functional tooth regeneration in swine. PLoS One. 2006; 1:e79.

12. Seo BM, Miura M, Gronthos S, et al. Investigation of multipotent postnatal stem cells from human periodontal ligament. Lancet. 2004;364(9429):149-55.

13. Morsczeck C, Gotz W, Schierholz J, et al. Isolation of precursor cells (PCs) from human dental follicle of wisdom teeth. Matrix Biol. 2005;24(2):155-65.

14.• Zhang Q, Shi S, Liu Y, et al. Mesenchymal stem cells derived from human gingiva are capable of immunomodulatory functions and ameliorate inflammation-related tissue destruction in experimental colitis. J Immunol. 2009;183(12):7787-98. This study firstly demonstrates both the in vitro and in vivo immunomodulatory functions of human gingiva-derived MSCs.

15. Gronthos S, Brahim J, Li W, et al. Stem cell properties of human dental pulp stem cells. J Dent Res. 2002;81(8):531-5.

16. Yu J, He H, Tang C, et al. Differentiation potential of STRO-1+ dental pulp stem cells changes during cell passaging. BMC Cell Biol. 2010;11:32.

17. Zhang W, Walboomers XF, Van Kuppevelt TH, et al. In vivo evaluation of human dental pulp stem cells differentiated towards multiple lineages. J Tissue Eng Regen Med. 2008;2(2-3):117-25.

18. Shi S, Bartold PM, Miura M, et al. The efficacy of mesenchymal stem cells to regenerate and repair dental structures. Orthod Craniofacial Res. 2005;8(3):191-9.

19. Batouli S, Miura M, Brahim J, et al. Comparison of stem-cellmediated osteogenesis and dentinogenesis. J Dent Res. 2003;82 (12):976-81.

20. Almushayt A, Narayanan K, Zaki AE, George A. Dentin matrix protein 1 induces cytodifferentiation of dental pulp stem cells into odontoblasts. Gene Ther. 2006;13(7):611-20.

21. He H, Yu J, Liu Y, et al. Effects of FGF2 and TGFbeta1 on the differentiation of human dental pulp stem cells in vitro. Cell Biol Int. 2008;32(7):827-34.

22. Mao JJ, Kim SG, Zhou J, et al. Regenerative endodontics: barriers and strategies for clinical translation. Dent Clin N Am. 2012;56(3): 639-49.

23. Huang GT. Dental pulp and dentin tissue engineering and regeneration: advancement and challenge. Front Biosci (Elite Ed). 2011;3: 788-800.

24. Huang GT, Yamaza T, Shea LD, et al. Stem/progenitor cellmediated de novo regeneration of dental pulp with newly deposited continuous layer of dentin in an in vivo model. Tissue Eng A. 2010;16(2):605-15. 
25. Iohara K, Imabayashi K, Ishizaka R, et al. Complete pulp regeneration after pulpectomy by transplantation of $\mathrm{CD} 105+$ stem cells with stromal cell-derived factor-1. Tissue Eng A. 2011;17(15-16): 1911-20. This study firstly demonstrates the potential for the complete pulp regeneration.

26. Iohara $\mathrm{K}$, Murakami $\mathrm{M}$, Takeuchi $\mathrm{N}$, et al. A novel combinatorial therapy with pulp stem cells and granulocyte colony-stimulating factor for total pulp regeneration. Stem Cells Trans1 Med. 2013;2(7):521-33

27. Alongi DJ, Yamaza T, Song Y, et al. Stem/progenitor cells from inflamed human dental pulp retain tissue regeneration potential. Regen Med. 2010;5(4):617-31.

28. Wang Z, Pan J, Wright JT, et al. Putative stem cells in human dental pulp with irreversible pulpitis: an exploratory study. J Endod. 2010;36(5):820-5.

29. Pereira LO, Rubini MR, Silva JR, et al. Comparison of stem cell properties of cells isolated from normal and inflamed dental pulps. Int Endod J. 2012;45(12):1080-90.

30. Ma D, Gao J, Yue J, et al. Changes in proliferation and osteogenic differentiation of stem cells from deep caries in vitro. J Endod. 2012;38(6):796-802.

31. Ji YM, Jeon SH, Park JY, et al. Dental stem cell therapy with calcium hydroxide in dental pulp capping. Tissue Eng A. 2010;16 (6): 1823-33

32. Yamada $\mathrm{Y}$, Ito $\mathrm{K}$, Nakamura $\mathrm{S}$, Ueda $\mathrm{M}$, Nagasaka T. Promising cell-based therapy for bone regeneration using stem cells from deciduous teeth, dental pulp, and bone marrow. Cell Transplant. 2011;20(7):1003-13.

33. d'Aquino R, De Rosa A, Lanza V, et al. Human mandible bone defect repair by the grafting of dental pulp stem/progenitor cells and collagen sponge biocomplexes. Eur Cell Mater. 2009;18:75-83.

34. Gandia C, Arminan A, Garcia-Verdugo JM, et al. Human dental pulp stem cells improve left ventricular function, induce angiogenesis, and reduce infarct size in rats with acute myocardial infarction. Stem Cells. 2008;26(3):638-45.

35. Kerkis I, Ambrosio CE, Kerkis A, et al. Early transplantation of human immature dental pulp stem cells from baby teeth to golden retriever muscular dystrophy (GRMD) dogs: Local or systemic? J Transl Med. 2008;6:35.

36. Monteiro BG, Serafim RC, Melo GB, et al. Human immature dental pulp stem cells share key characteristic features with limbal stem cells. Cell Prolif. 2009;42(5):587-94.

37. Gomes JA, Geraldes Monteiro B, Melo GB, et al. Corneal reconstruction with tissue-engineered cell sheets composed of human immature dental pulp stem cells. Investig Ophthalmol Vis Sci. 2010;51(3):1408-14.

38. Kiraly M, Kadar K, Horvathy DB, et al. Integration of neuronally predifferentiated human dental pulp stem cells into rat brain in vivo. Neurochem Int. 2011;59(3):371-81.

39. Sugiyama M, Iohara K, Wakita H, et al. Dental pulp-derived CD31 $(-) / \mathrm{CD} 146(-)$ side population stem/progenitor cells enhance recovery of focal cerebral ischemia in rats. Tissue Eng A. 2011;17(9-10): 1303-11

40. Kerkis I, Kerkis A, Dozortsev D, et al. Isolation and characterization of a population of immature dental pulp stem cells expressing OCT-4 and other embryonic stem cell markers. Cells Tissues Organs. 2006;184(3-4):105-16.

41. Sakai VT, Zhang Z, Dong Z, et al. SHED differentiate into functional odontoblasts and endothelium. J Dent Res. 2010;89(8):791-6.

42. Casagrande L, Demarco FF, Zhang Z, et al. Dentin-derived BMP-2 and odontoblast differentiation. J Dent Res. 2010;89(6):603-8.

43. Cordeiro MM, Dong Z, Kaneko T, et al. Dental pulp tissue engineering with stem cells from exfoliated deciduous teeth. J Endod. 2008;34(8):962-9.

44. Nakamura S, Yamada Y, Katagiri W, et al. Stem cell proliferation pathways comparison between human exfoliated deciduous teeth and dental pulp stem cells by gene expression profile from promising dental pulp. J Endod. 2009;35(11):1536-42.

45. Koyama N, Okubo Y, Nakao K, Bessho K. Evaluation of pluripotency in human dental pulp cells. J Oral Maxillofac Surg. 2009;67(3):501-6.

46. Gotlieb EL, Murray PE, Namerow KN, Kuttler S, Garcia-Godoy F. An ultrastructural investigation of tissue-engineered pulp constructs implanted within endodontically treated teeth. J Am Dent Assoc. 2008;139(4):457-65.

47. Seo BM, Sonoyama W, Yamaza T, et al. SHED repair critical-size calvarial defects in mice. Oral Dis. 2008;14(5):428-34.

48. Zheng Y, Liu Y, Zhang CM, et al. Stem cells from deciduous tooth repair mandibular defect in swine. J Dent Res. 2009;88(3):249-54.

49. Govindasamy V, Abdullah AN, Ronald VS, et al. Inherent differential propensity of dental pulp stem cells derived from human deciduous and permanent teeth. J Endod. 2010;36(9):1504-15.

50. Wang J, Wang X, Sun Z, et al. Stem cells from human-exfoliated deciduous teeth can differentiate into dopaminergic neuron-like cells. Stem Cells Dev. 2010;19(9):1375-83.

51. Huang GT, Sonoyama W, Liu Y, et al. The hidden treasure in apical papilla: the potential role in pulp/dentin regeneration and bioroot engineering. J Endod. 2008;34(6):645-51.

52. Bakopoulou A, Leyhausen G, Volk J, et al. Comparative analysis of in vitro osteo/odontogenic differentiation potential of human dental pulp stem cells (DPSCs) and stem cells from the apical papilla (SCAP). Arch Oral Biol. 2011;56(7):709-21.

53. Abe S, Yamaguchi S, Watanabe A, Hamada K, Amagasa T. Hard tissue regeneration capacity of apical pulp derived cells (APDCs) from human tooth with immature apex. Biochem Biophys Res Commun. 2008;371(1):90-3.

54. Huang GT, Gronthos S, Shi S. Mesenchymal stem cells derived from dental tissues vs. those from other sources: their biology and role in regenerative medicine. J Dent Res. 2009;88(9):792-806.

55. Friedlander LT, Cullinan MP, Love RM. Dental stem cells and their potential role in apexogenesis and apexification. Int Endod J. 2009;42(11):955-62.

56. Gay IC, Chen S, MacDougall M. Isolation and characterization of multipotent human periodontal ligament stem cells. Orthod Craniofacial Res. 2007;10(3):149-60.

57. Lindroos B, Maenpaa K, Ylikomi T, et al. Characterisation of human dental stem cells and buccal mucosa fibroblasts. Biochem Biophys Res Commun. 2008;368(2):329-35.

58. Xu J, Wang W, Kapila Y, Lotz J, Kapila S. Multiple differentiation capacity of STRO-1+/CD146+ PDL mesenchymal progenitor cells. Stem Cells Dev. 2009;18(3):487-96.

59. Techawattanawisal W, Nakahama K, Komaki M, et al. Isolation of multipotent stem cells from adult rat periodontal ligament by neurosphere-forming culture system. Biochem Biophys Res Commun. 2007;357(4):917-23.

60. Sonoyama W, Seo BM, Yamaza T, Shi S. Human Hertwig's epithelial root sheath cells play crucial roles in cementum formation. J Dent Res. 2007;86(7):594-9.

61. Liu Y, Zheng Y, Ding G, et al. Periodontal ligament stem cellmediated treatment for periodontitis in miniature swine. Stem Cells. 2008;26(4):1065-73.

62. Park JY, Jeon SH, Choung PH. Efficacy of periodontal stem cell transplantation in the treatment of advanced periodontitis. Cell Transplant. 2011;20(2):271-85.

63. Feng F, Akiyama K, Liu Y, et al. Utility of PDL progenitors for in vivo tissue regeneration: a report of 3 cases. Oral Dis. 2010;16(1): 20-8. This work describes the potential for the application of PDLSCs in the reconstruction of periodontal defects in human clinical study.

64. Lin Y, Gallucci GO, Buser D, et al. Bioengineered periodontal tissue formed on titanium dental implants. J Dent Res. 2011;90 (2):251-6 
65. Kim SH, Kim KH, Seo BM, et al. Alveolar bone regeneration by transplantation of periodontal ligament stem cells and bone marrow stem cells in a canine peri-implant defect model: a pilot study. $\mathrm{J}$ Periodontol. 2009;80(11):1815-23.

66. Gault P, Black A, Romette JL, et al. Tissue-engineered ligament: implant constructs for tooth replacement. J Clin Periodontol. 2010;37(8):750-8.

67. Yao S, Pan F, Prpic V, Wise GE. Differentiation of stem cells in the dental follicle. J Dent Res. 2008;87(8):767-71.

68. Guo W, Gong K, Shi H, et al. Dental follicle cells and treated dentin matrix scaffold for tissue engineering the tooth root. Biomaterials. 2012;33(5):1291-302.

69. Handa K, Saito M, Tsunoda A, et al. Progenitor cells from dental follicle are able to form cementum matrix in vivo. Connect Tissue Res. 2002;43(2-3):406-8.

70. Handa K, Saito M, Yamauchi M, et al. Cementum matrix formation in vivo by cultured dental follicle cells. Bone. 2002;31(5):606-11.

71. Honda MJ, Imaizumi M, Suzuki H, et al. Stem cells isolated from human dental follicles have osteogenic potential. Oral Surg Oral Med Oral Pathol Oral Radiol Endod. 2011;111(6):700-8.

72. Angiero F, Rossi C, Ferri A, et al. Stromal phenotype of dental follicle stem cells. Front Biosci (Elite Ed). 2012;4:1009-14.

73. Yokoi T, Saito M, Kiyono T, et al. Establishment of immortalized dental follicle cells for generating periodontal ligament in vivo. Cell Tissue Res. 2007;327(2):301-11.

74. Yagyuu T, Ikeda E, Ohgushi H, et al. Hard tissue-forming potential of stem/progenitor cells in human dental follicle and dental papilla. Arch Oral Biol. 2010;55(1):68-76.

75. Morsczeck C, Vollner F, Saugspier M, et al. Comparison of human dental follicle cells (DFCs) and stem cells from human exfoliated deciduous teeth (SHED) after neural differentiation in vitro. Clin Oral Investig. 2010;14(4):433-40.

76. Irwin CR, Picardo M, Ellis I, et al. Inter- and intra-site heterogeneity in the expression of fetal-like phenotypic characteristics by gingival fibroblasts: potential significance for wound healing. J Cell Sci. 1994;107(Pt 5):1333-46.

77. Stephens P, Davies KJ, Occleston N, et al. Skin and oral fibroblasts exhibit phenotypic differences in extracellular matrix reorganization and matrix metalloproteinase activity. Br J Dermatol. 2001;144(2): 229-37.

78. Tang L, Li N, Xie H, Jin Y. Characterization of mesenchymal stem cells from human normal and hyperplastic gingiva. J Cell Physiol. 2011;226(3):832-42.

79. Tomar GB, Srivastava RK, Gupta N, et al. Human gingiva-derived mesenchymal stem cells are superior to bone marrow-derived mesenchymal stem cells for cell therapy in regenerative medicine. Biochem Biophys Res Commun. 2010;393(3):377-83.

80. Wang F, Yu M, Yan X, et al. Gingiva-derived mesenchymal stem cell-mediated therapeutic approach for bone tissue regeneration. Stem Cells Dev. 2011;20(12):2093-102.

81. Marynka-Kalmani K, Treves S, Yafee M, et al. The lamina propria of adult human oral mucosa harbors a novel stem cell population. Stem Cells. 2010;28(5):984-95.

82. Fournier BP, Ferre FC, Couty L, et al. Multipotent progenitor cells in gingival connective tissue. Tissue Eng A. 2010;16(9):2891-9.

83. Ding G, Wang W, Liu Y, et al. Effect of cryopreservation on biological and immunological properties of stem cells from apical papilla. J Cell Physiol. 2010;223(2):415-22.

84. Zhang Q, Nguyen AL, Shi S, et al. Three-dimensional spheroid culture of human gingiva-derived mesenchymal stem cells enhances mitigation of chemotherapy-induced oral mucositis. Stem Cells Dev. 2012;21(6):937-47.

85. Lee RH, Oh JY, Choi H, Bazhanov N. Therapeutic factors secreted by mesenchymal stromal cells and tissue repair. J Cell Biochem. 2011;112(11):3073-8.

86. Prockop DJ, Oh JY. Mesenchymal stem/stromal cells (MSCs): role as guardians of inflammation. Mol Ther. 2012;20(1):14-20.

87. Su WR, Zhang QZ, Shi SH, Nguyen AL, Le AD. Human gingivaderived mesenchymal stromal cells attenuate contact hypersensitivity via prostaglandin E2-dependent mechanisms. Stem Cells. 2011;29(11):1849-60.

88. Chen M, Su W, Lin X, et al. Adoptive transfer of human gingivaderived mesenchymal stem cells ameliorates collagen-induced arthritis via suppression of Th1 and Th17 cells and enhancement of regulatory T cell differentiation. Arthritis Rheum. 2013;65(5): 1181-93. 